Review Article

\title{
Excited State Proton Transfer of a Versatile Fluorescent Probe in Different Reverse Micelles: An Overview
}

\author{
SHARMISTHA DUTTA CHOUDHURY ${ }^{1,2, *}$ and HARIDAS PAL ${ }^{1,2}$ \\ ${ }^{1}$ Radiation \& Photochemistry Division, Bhabha Atomic Research Centre, Mumbai 400 085, India \\ ${ }^{2}$ Homi Bhabha National Institute, Training School Complex, Anushaktinagar, Mumbai 400 094, India
}

(Received on 21 March 2018; Accepted on 15 September 2018)

\begin{abstract}
Proton transfer reactions are ubiquitous in chemical and biological systems. In this article we describe the excited state prototropic behavior of 7-hydroxy-4-methyl coumarin (7H4MC) in different kinds of reverse micelles. The dye, 7H4MC, is interesting because depending on the solvent conditions it can exist in the neutral, anionic or tautomeric forms, in its excited state. These prototropic forms have characteristic emission spectral features, which makes 7H4MC an excellent fluorescence reporter of aqueous microenvironments. In nonpolar solvents, 7H4MC exists in the neutral form in both the ground and the excited states. In aqueous medium, the dye exists in the neutral form in the ground state but is converted quantitatively to the anionic form in the excited state. In ionic reverse micelles, both the anionic and tautomeric forms evolve simultaneously from the excited neutral form of the dye, with increasing $\mathrm{w}_{0}$ values $\left(\mathrm{w}_{0}=\left[\mathrm{H}_{2} \mathrm{O}\right] /[\right.$ surfactant $\left.]\right)$. However, the anion form is more favoured in cationic reverse micelles compared to anionic reverse micelles, due to additional electrostatic stabilization of the anion in the former system. In nonionic reverse micelles, although both anionic and tautomeric forms of the dye are observed on increasing the $\mathrm{w}_{0}$ values, their contributions are quite low due to the absence of a well-defined water pool in these systems. The excited state prototropism of 7H4MC in aqueous mixtures of 1-alkyl-3-methylimidazolium ionic liquids (ILs) resembles that in the conventional cationic reverse micelle systems, and reveals that reverse micelle-like water pools are formed in IL-water mixtures as well.
\end{abstract}

Keywords: Prototropism; Tautomer; Fluorescence; Reverse Micelle; Ionic Liquid; Water Pool

\section{Introduction}

Proton transfer is one of the most common and fundamental reactions that plays important roles in a wide variety of biological and chemical phenomena. In many cases, proton transfer is found to be more favourable in the excited state than in the ground state, due to a modulation in the acid-base characteristics of the molecules upon their electronic excitation (Arnaut and Formosinho, 1993; Formosinho and Arnaut, 1993). This leads to interesting applications of proton transfer molecules in diverse areas like, photolithography, laser dyes, sensors and optical switches (Kwon and Park, 2011; Zhao et al., 2012). Therefore, excited state proton transfer reactions are extensively investigated in different homogeneous as well as microheterogenous media, for a fundamental understanding and for their various applications.
Many different kinds of excited state proton transfer reactions are encountered in the literature (Bardez, 1999; Waluk, 2003). In monofunctional molecules, $\mathrm{RH}$, that possess a proton donating group, excitation often leads to the creation of a stronger photoacid, i.e., $\mathrm{pK}_{\mathrm{a}}^{*}\left(\mathrm{RH}^{*} / \mathrm{R}^{-*}\right)<<\mathrm{pK}_{\mathrm{a}}\left(\mathrm{RH} / \mathrm{R}^{-}\right)$. As a result, photoinduced intermolecular proton transfer to the surrounding solvent molecules becomes quite facile, leading to the generation of the anion, $\mathrm{R}^{*}$. One such prototypical photoacid molecule is pyranine or 8-hydroxypyrene-1,3,6-trisulfonic acid (Chart 1A) (Siwick and Bakker, 2007). In bifunctional molecules that possess both proton donating and proton accepting functionalities, an increase in the acidities and basicities of the functional groups can occur in the excited state. This facilitates intramolecular proton transfer from one molecular site to another, leading to the generation of prototropic tautomers. The simplest situation arises

*Author for Correspondence: E-mail: sharmidc@barc.gov.in 


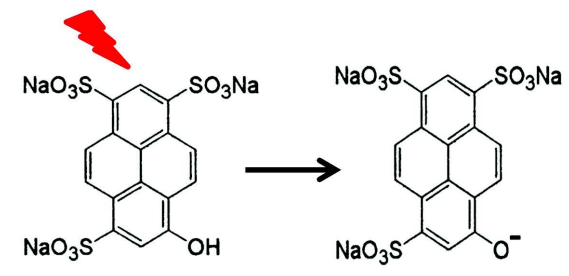

(A) Intermolecular proton transfer

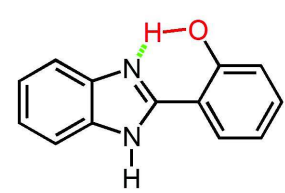

(B) Vicinal

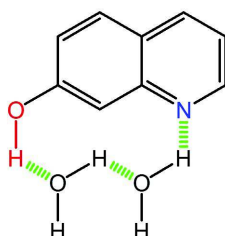

(C) Solvent mediated

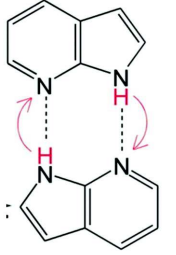

(D) Biprotonic transfer
Intramolecular proton transfer

Chart 1: Different kinds of excited state proton transfer processes. (A) intermolecular proton transfer in pyranine, (B) Direct intramolecular proton transfer in 2-(2'-hydroxyphenyl) benzimidazole, (C) Solvent mediated intramolecular proton transfer in 7 hydroxyquinoline, (D) Biprotonic transfer in 7azaindole

when the proton donating and accepting groups are in close proximity and H-bonded to each other. In this case, the proton transfer occurs directly through the pre-existing intramolecular H-bond, as depicted for the representative molecule, 2-(2'-hydroxyphenyl) benzimidazole, in Chart 1B (Kwon and Park, 2011). Alternatively, the proton donating and accepting substituents may be distal, in which case the proton relocation is mediated through solvent $\mathrm{H}$-bonded bridges, as depicted for 7-hydroxyquinoline, Chart 1C (Waluk, 2003). Tautomerization can also occur through double proton transfer within doubly $\mathrm{H}$-bonded dimers of the bifunctional molecules. The most well studied molecule that depicts photoinduced biprotonic transfer is 7-azaindole, Chart 1D (Takeuchi and Tahara, 2007).

Since proton transfer is extremely sensitive to the structure and dynamics of the surrounding water molecules, these reactions can be used as convenient reporters of the solvent microenvironment. In fact, studies on excited state prototropic behavior of fluorescent molecules have led to a wealth of knowledge about the microenvironments in micelles, reverse micelles, biological cells, proteins, macrocyclic hosts, membrane films and other supramolecular structures (Amdursky et al., 2014; Chowdury et al.,
2015; Cohen et al., 2011; Douhal et al., 2007; Gepshtein et al., 2006; Singhal et al., 2016).

In this article, we focus especially on reverse micellar structures, which are spherical aggregates of surfactants dispersed in a nonpolar medium. Typically, the reverse micelles consist of an inner water pool surrounded by a layer of surfactant molecules having their polar head groups oriented toward the inner water pool and the hydrocarbon chains toward the bulk nonpolar medium (Jain et al., 1989; Kalyanasundaram, 1987; McNeil and Thomas, 1981; Luisi, 1988). The average size of the reverse micelles depend on the amount of solubilized water and is generally related to the water to surfactant molar ratio, $\mathrm{w}_{0}=\left[\mathrm{H}_{2} \mathrm{O}\right] /[$ surfactant $]$. It is quite well established that the properties of the water encapsulated inside the reverse micelles differ significantly from those of bulk water. At least two types of water populations are expected inside the water pool; a shell of "bound" water molecules that are associated with the polar head groups of the surfactants through H-bonding or ion-dipole interactions, and the "free" water molecules that develop in the core of the water pool with increasing $\mathrm{w}_{0}$ (Agazzi et al., 2013; Blach et al., 2011; Moilanen et al., 2007; Piletic et al., 2006). Reverse micelles are often used as simple bio-mimicking assemblies to understand the properties of confined water in real biological systems. In this regard, it is interesting to compare and analyze the excited state proton transfer reactions of a prototropic molecule solubilized in different kinds of reverse micelles, and hence gain some insight about the nature of their water pools. With this perspective, we discuss below, the excited state prototropism of a versatile probe dye in ionic and nonionic reverse micelles formed by conventional surfactants, namely, the anionic surfactant, sodium 1,4-bis(2-ethylhexyl)sulfosuccinate (AOT), the cationic surfactant, benzyldimethylhexadecylammonium chloride (BHDC), and the neutral surfactant, poly(oxyethylene)(tetramethylbutyl)phenyl ether (TritonX-100 or TX-100). We also discuss excited state proton transfer in nonconventional reverse micelle-like structures formed in the aqueous mixtures of surface active ionic liquids (ILs) composed of 1-alkyl-3-methylimidazolium cations $\left(\mathrm{C}_{\mathrm{n}} \mathrm{mim}\right)$ with varying alkyl chain lengths $(\mathrm{n}=2,4,6,8$ and 10$)$, and having tetrafluoroborate, $\left[\mathrm{BF}_{4}\right]^{-}$, as the anion. The structures of the conventional surfactants and the ILs 
are presented in Chart 2. A schematic of the reverse micelle structures formed by conventional surfactants and in the IL-water systems, are presented in Chart 3 .

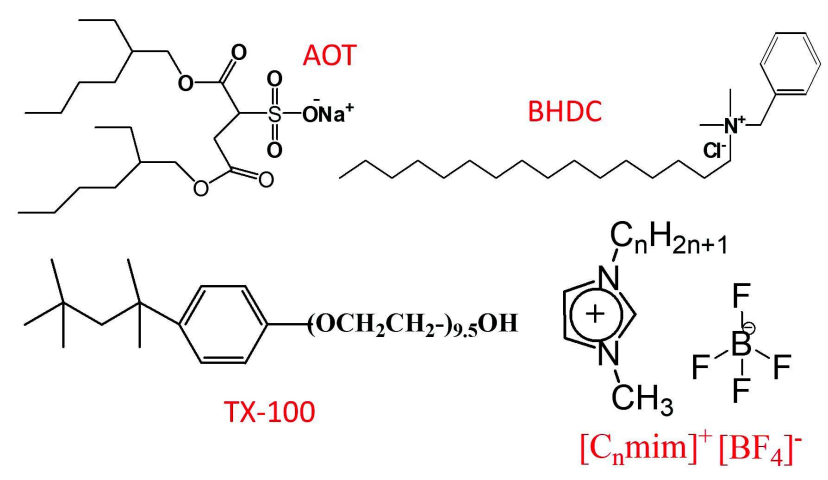

Chart 2: Structures of conventional ionic (AOT, BHDC) and nonionic (TX-100) surfactants, and the surface active ionic liquids $\left(\left[\mathrm{C}_{\mathrm{n}} \mathrm{mim}\right]\left[\mathrm{BF}_{4}\right]\right)$ discussed in the present study
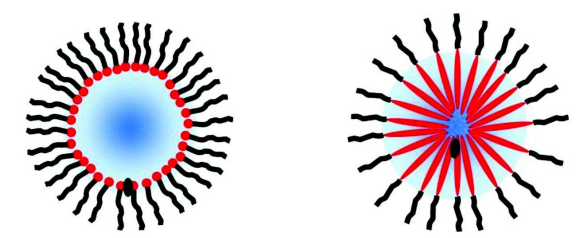

(A) lonic reverse micelle, (B) Nonionic reverse micelle

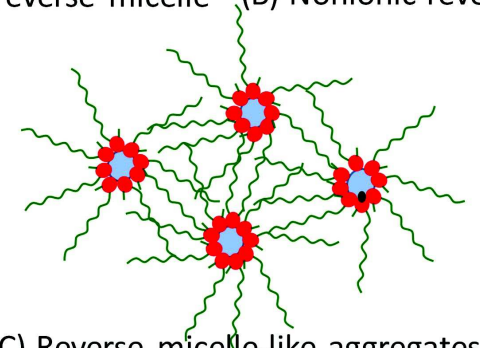

(C) Reverse micelle-like aggregates

Chart 3: Schematic representation of different reverse micelle structures; (A) AOT/BHDC, (B) TX-100, (C) IL-water mixtures

\section{Prototropism of 7-hydroxy-4-methylcoumarin (7H4MC)}

The coumarin derivative, 7-hydroxy-4methylcoumarin (7H4MC), is an interesting prototropic dye, because depending on the $\mathrm{pH}$ of the medium and the solvent structure, it can undergo both intermolecular proton transfer giving rise to the anion form, as well as solvent mediated intramolecular proton transfer giving rise to the tautomer form (AbdelMottaleb et al., 1989; Bardez et al., 1992; de Melo and Maçanita 1993; Dutta Choudhury et al., 2008;

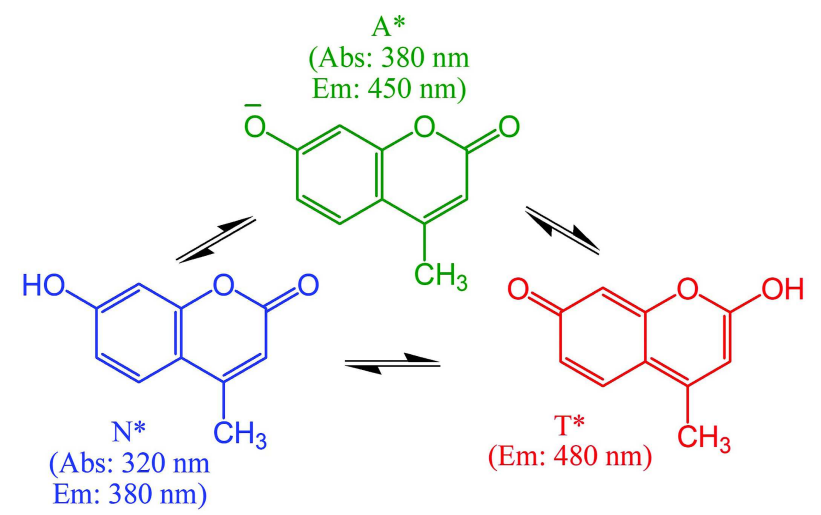

Chart 4: Excited state prototropic forms of 7H4MC (Neutral: $\mathrm{N}^{*}$, Anion: $\mathrm{A}^{*}$, Tautomer: $\mathrm{T}^{*}$ )

Dutta Choudhury and Pal, 2009; Moriya, 1983; Simkovitch et al., 2016). The prototropic transformations of 7H4MC are depicted in Chart 4. In the ground state, the dye can exist in the neutral or anionic form, at suitable $\mathrm{pH}$ conditions. The ground state $\mathrm{pK}_{\mathrm{a}}$ value of $7 \mathrm{H} 4 \mathrm{MC}$ is about 7.7. On photoexcitation, however, its acidity increases quite sharply $\left(\mathrm{pK}_{\mathrm{a}}^{*} \sim 0.45\right)$, thus favoring the formation of the anion in the excited state. The tautomeric form arises in the excited state due to water mediated proton transfer between the spatially separated proton donor $(\mathrm{OH})$ and acceptor $(\mathrm{C}=\mathrm{O})$ groups of 7H4MC (DeSilva et al., 2013; Georgieva et al., 2007). Importantly, each of the prototropic forms of 7H4MC have characteristic absorption/emission features, which are quite useful for the identification of the different species, and hence for the determination of the extent of prototropic transformations taking place in any particular solvent condition. Depending on the solvent environment, the fluorescence spectrum of 7H4MC can be composed of emissions from the excited neutral from $\left(\mathrm{N}^{*} \sim 380\right.$ $\mathrm{nm})$, anionic form $\left(\mathrm{A}^{*} \sim 450 \mathrm{~nm}\right)$ and/or the tautomeric form ( $\mathrm{T}^{*} \sim 480 \mathrm{~nm}$ ) (Abdel-Mottaleb et al., 1989; Bardez et al., 1992; de Melo and Maçanita., 1993; Dutta Choudhury, et al., 2008; Dutta Choudhury and Pal, 2009; Moriya, 1983; Simkovitch et al., 2016). The quantum yields of the three species are reported to be $0.62,0.85$ and 0.74 , respectively (de Melo and Maçanita., 1993). The typical absorption and emission features of $7 \mathrm{H} 4 \mathrm{MC}$ in a representative nonpolar solvent, dioxane, in water and in dioxane-water mixture (30\% v/v water) are presented in Fig. 1 for a clear understanding. The absorption spectrum of the dye in dioxane and in water has maximum around 
$320 \mathrm{~nm}$, which corresponds to the neutral form (N) of the dye. In the nonpolar solvent, the emission also corresponds to the neutral form $\left(\mathrm{N}^{*}\right)$, with maximum around $380 \mathrm{~nm}$. However, in water the emission maximum is significantly shifted to $450 \mathrm{~nm}$, corresponding predominantly to the anionic form $\left(\mathrm{A}^{*}\right)$ of the dye. In dioxane-water mixture ( $30 \% \mathrm{v} / \mathrm{v}$ water), the emission spectrum shows two clear bands, one at $380 \mathrm{~nm}$ corresponding to $\mathrm{N}^{*}$ and another around 480 $\mathrm{nm}$, corresponding to the tautomer form, $\mathrm{T}^{*}$.

Quite understandably, the multiple optical signatures of $7 \mathrm{H} 4 \mathrm{MC}$ can serve as valuable spectroscopic reporters of the microenvironment. Hence this dye was considered quite suitable for investigating different reverse micelle systems formed by AOT, BHDC, TX100 or ILs (Dutta Choudhury et al., 2008; Dutta Choudhury and Pal, 2009). Our studies have revealed that the probe preferably resides in the interfacial region of the reverse micelles, near the boundary separating the water pool from the relatively nonpolar medium composed of the alkyl chains of the surfactants and the nonpolar solvent. Accordingly, small changes in the nature of the reverse micelles and the water pool environments is directly reflected in the excited state proton transfer processes of 7H4MC. It may be mentioned that following our initial studies on the excited state proton transfer of $7 \mathrm{H} 4 \mathrm{MC}$ in reverse micelles, the interesting prototropic transformation of this dye was also used by Amaro et al. to probe site-specific hydration in haloalkane dehalogenase enzymes (Amaro et al., 2015).

\section{Effect of Ionic Reverse Micelles on the Excited State Prototropism of $7 \mathrm{H} 4 \mathrm{MC}$}

Figures 2A and 2B depict the emission spectra of 7H4MC in AOT/n-heptane/water and BHDC/ benzene/water reverse micelle systems, respectively, at different $\mathrm{w}_{0}$ values. In both the anionic and the cationic reverse micelles, there is one emission band around $380 \mathrm{~nm}$, corresponding to the neutral form, $\mathrm{N}^{*}$, in the absence of added water $\left(\mathrm{w}_{0}=0\right)$. It may be mentioned that the absorption spectra of 7H4MC in both AOT and BHDC reverse micelles at $\mathrm{w}_{0}=0$, also show a single band with maximum around $320 \mathrm{~nm}$ corresponding to the absorption of the neutral form, (as also observed for the dye in dioxane and bulk water, cf. Fig. 1). Interestingly, although the absorption spectra remain unchanged with increasing $\mathrm{w}_{0}$ values

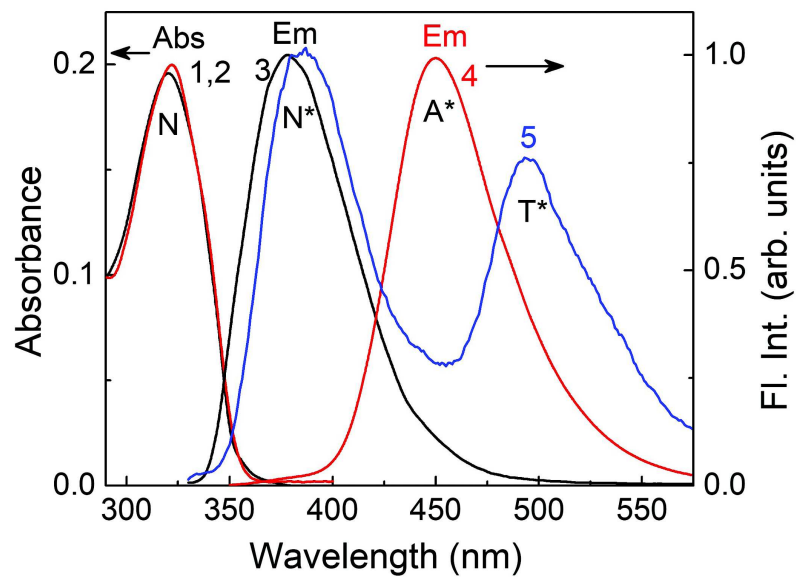

Fig. 1: Absorption spectra of 7H4MC in dioxane (1) and water (2); and emission spectra of 7H4MC in dioxane (3), water (4) and dioxane water mixture $(30 \% \mathrm{v} / \mathrm{v}$ water) (5). Excitation wavelength is $320 \mathrm{~nm}$. Species responsible for different bands are indicated in the figure

in the reverse micelles, the emission spectral characteristics of the dye show significant changes. As indicated from Fig. 2, with increase in the water content, there is a gradual decrease in the emission from the neutral form $(380 \mathrm{~nm})$ along with the simultaneous appearance of emission from the anionic $(\sim 450 \mathrm{~nm})$ and the tautomeric $(\sim 480 \mathrm{~nm})$ forms of the dye. A distinct isoemissive point is observed in both the reverse micelle systems. These results clearly suggest that though only the neutral form of the dye exists in the ground state in AOT and BHDC reverse micelle systems, an increase in the water pool size of the reverse micelles facilitates the transformation of the excited neutral form of the dye to both the anion as well as the tautomer forms. The formation of both the anionic and tautomeric forms of 7H4MC in the interfacial region of the reverse micelle water pools, as opposed to the formation of predominantly anionic species in bulk water (cf. Fig. 1), suggests that in the confined environment of the reverse micellar interface, the preferential stabilization of the anionic form over the tautomeric form is not that substantial, certainly due to a reduction in the number of available water molecules and/or the partially broken hydrogen bonded network structure of water encapsulated within the reverse micelle (Dutta Choudhury et al., 2008; Dutta Choudhury and Pal, 2009).

It may be noted however, that while in AOT reverse micelles, the intensity ratios of the emission from $\mathrm{A}^{*}$ and $\mathrm{T}^{*}$ forms remain quite similar at different 

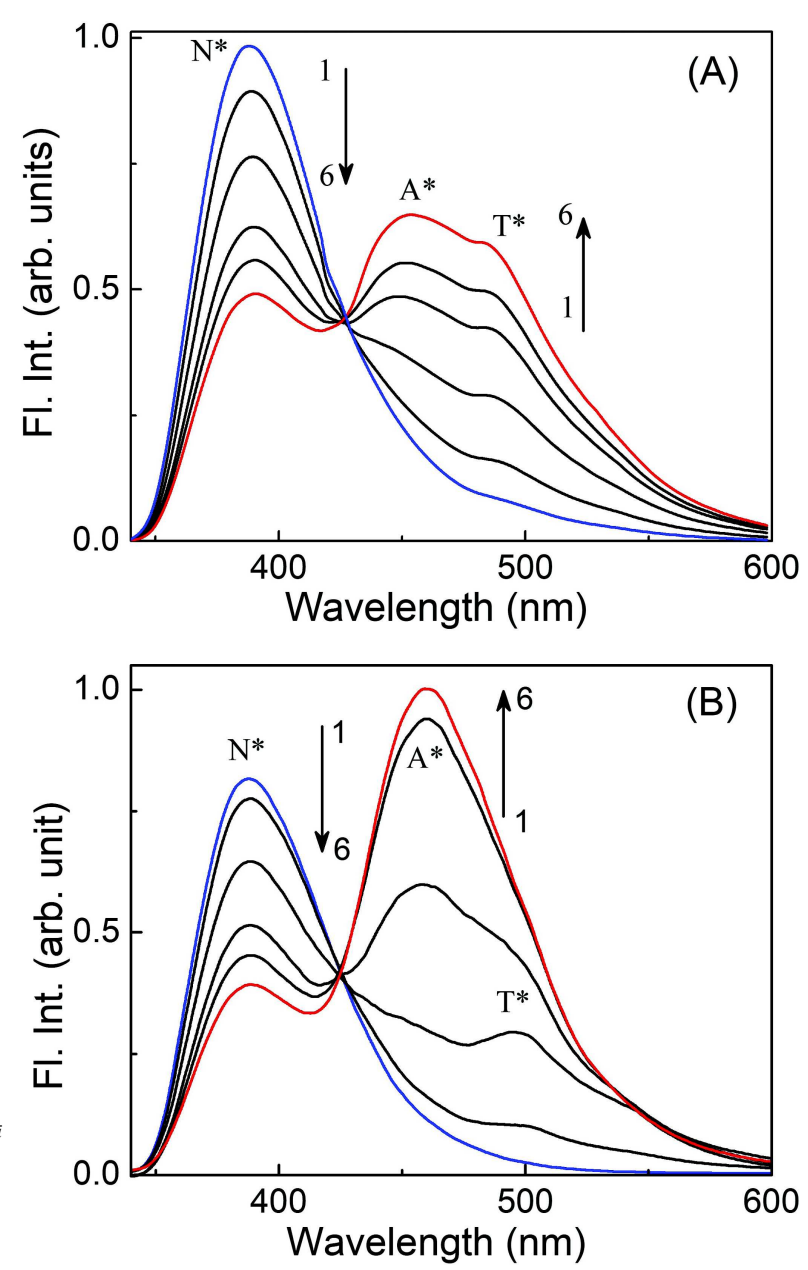

Fig. 2: (A) Emission spectra of $7 \mathrm{H} 4 \mathrm{MC}$ in AOT reverse micelles at $w_{0}$ values of $0,5,10,20,30$ and 60 (1-6). (B) Emission spectra of $7 \mathrm{H} 4 \mathrm{MC}$ in BHDC reverse micelles at $w_{0}$ values of $0,2,5,10,20$ and 30 (1-6). Species responsible for different bands are indicated in the figure. Excitation wavelength is $320 \mathrm{~nm}$. (Adapted with permission from Dutta Choudhury et al., 2008. Copyright 2008 American Chemical Society and Dutta Choudhury and Pal, 2009. Copyright 2009 American Chemical Society) $\mathrm{w}_{0}$ values (cf. Fig. 2A), in BHDC reverse micelles, the emission intensity of $\mathrm{A}^{*}$ gradually becomes higher than that of $\mathrm{T}^{*}$, with increasing $\mathrm{w}_{0}$ values (cf. Fig. 2B). These differences between the two reverse micelle systems indicate that the different charges of the head groups in AOT and BHDC surfactants have a significant effect on the excited state prototropic behavior of 7H4MC. At low $\mathrm{w}_{0}$ values, when most of the water molecules in the reverse micelles are in the bound form, the anionic form of the dye cannot be stabilized to a greater extent than the tautomer form. However, at higher values of $\mathrm{w}_{0}$, the amount of free water within the water pool increases, and consequently the solvation and stabilization of the anionic form is more efficient. In the BDHC reverse micelles, the anionic form can be further stabilized due to electrostatic interaction with the positively charged surfactant head groups. As a result, the excited neutral form of the dye is preferably converted to the anionic from than to the tautomer form, at high $\mathrm{w}_{0}$ values in BDHC reverse micelles (Dutta Choudhury and Pal, 2009).

The fluorescence decay parameters of 7H4MC monitored around $380 \mathrm{~nm}, 450 \mathrm{~nm}$ and $500 \mathrm{~nm}$, in both the reverse micelles at $\mathrm{w}_{0}$ values of 0 and 20 are presented in Table 1 and representative decay traces of the dye in AOT reverse micelles, at different emission wavelengths are presented in Fig. 3. It is clear that with the addition of water in the reverse micelles, there is a substantial decrease in the lifetime of the neutral form of the dye, along with the appearance of a growth component at the higher wavelengths corresponding to the emissions of the anionic and tautomeric forms. The longer decay components observed around $450 \mathrm{~nm}$ and $500 \mathrm{~nm}$ are attributed to the lifetimes of the $\mathrm{A}^{*}$ and $\mathrm{T}^{*}$ forms

Table 1: Decay parameters ${ }^{\mathrm{a}}$ of $7 \mathrm{H} 4 \mathrm{MC}$ in AOT and BHDC reverse micelles monitored at different wavelengths corresponding corresponding to the emission positions of the neutral, anionic and tautomeric forms of the dye

\begin{tabular}{|c|c|c|c|c|c|c|c|c|c|c|c|}
\hline \multirow[t]{2}{*}{ System } & \multirow{2}{*}{$\mathrm{w}_{0}$} & \multicolumn{2}{|c|}{$\sim 380 \mathrm{~nm}\left(\mathrm{~N}^{*}\right)$} & \multicolumn{4}{|c|}{$\sim 450 \mathrm{~nm}\left(\mathrm{~A}^{*}\right)$} & \multicolumn{3}{|c|}{$\sim 500 \mathrm{~nm}\left(\mathrm{~T}^{*}\right)$} & \multirow[b]{2}{*}{$\tau_{2}(\mathrm{~ns})$} \\
\hline & & $A_{1}(\%)$ & $\tau_{1}(\mathrm{~ns})$ & $\mathrm{A}_{1}(\%)$ & $\tau_{1}(\mathrm{~ns})$ & $\mathrm{A}_{2}(\%)$ & $\tau_{2}(\mathrm{~ns})$ & $\mathrm{A}_{1}(\%)$ & $\tau_{1}(\mathrm{~ns})$ & $\mathrm{A}_{2}(\%)$ & \\
\hline \multirow[t]{2}{*}{ AOT } & 0 & 100 & 2.3 & - & - & - & - & - & - & - & - \\
\hline & 20 & 100 & 1.2 & 105 & 4.9 & -5 & 1.1 & 120 & 5.0 & -20 & 1.1 \\
\hline \multirow[t]{2}{*}{ BHDC } & 0 & 100 & 1.4 & - & - & - & - & - & - & - & - \\
\hline & 20 & 100 & 1.0 & 100 & 4.7 & - & - & 110 & 4.5 & -10 & 1.0 \\
\hline
\end{tabular}

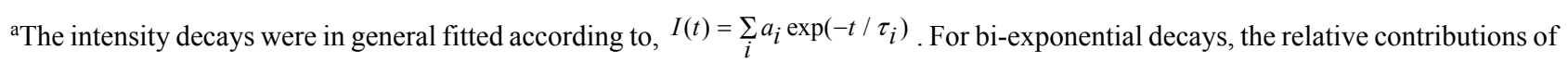




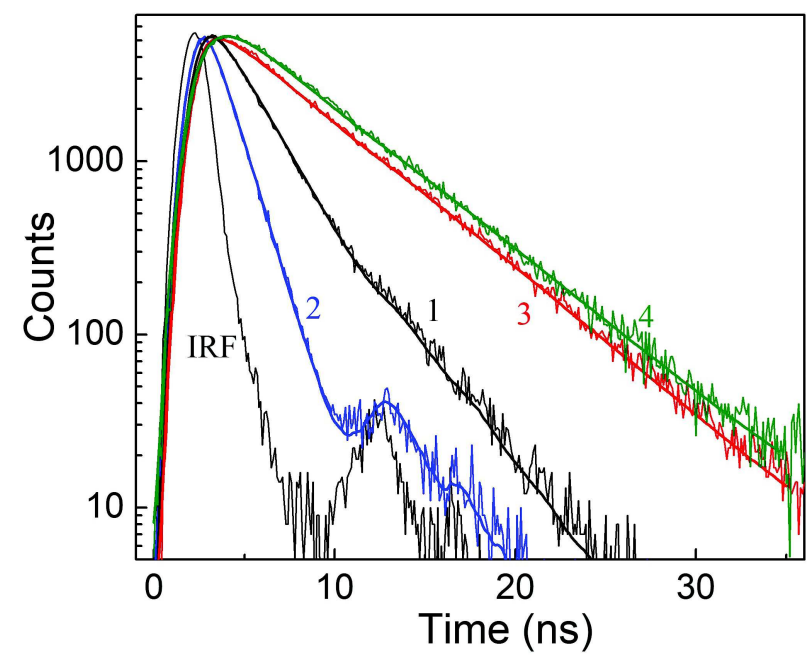

Fig. 3: Fluorescence decay traces of $7 \mathrm{H} 4 \mathrm{MC}$ in $\mathrm{AOT}$ reverse micelles monitored at (1) $380 \mathrm{~nm}, w_{0}=0$, (2) $380 \mathrm{~nm}$, $\mathrm{w}_{0}=20$, (3) $450 \mathrm{~nm}, \mathrm{w}_{0}=20$ and (4) $530 \mathrm{~nm}, \mathrm{w}_{0}=20$. IRF is the instrument response function, excitation wavelength is $320 \mathrm{~nm}$. (Adapted with permission from Dutta Choudhury et al., 2008. Copyright 2008 American Chemical Society)

of 7H4MC, respectively, in the corresponding reverse micelle systems (Dutta Choudhury et al., 2008; Dutta Choudhury and Pal, 2009).

\section{Effect of Nonionic Reverse Micelles on the Excited State Prototropism of 7H4MC}

Unlike the ionic surfactants that have a localized polar head group, the nonionic surfactant TX-100 possesses an extended hydrophilic oxyethylene chain of average 9.5 units, which is longer than the hydrophobic part. The amount of water solubilized in the nonionic reverse micelles is substantially low compared to the ionic reverse micelles (Dutt 2004, Zhu et al., 1992; Zhu and Schelly 1992). In the studied TX-100/benzenehexane/water reverse micelle system, phase separation is observed beyond $\mathrm{w}_{0} \sim 9.5$ (Dutta Choudhury and Pal, 2009). Furthermore, unlike the spherical and well demarcated water pools of ionic reverse micelles, the water pool in nonionic reverse micelles is not well-defined (cf. Chart 3). The water is mainly dissolved in the regions of the TX-100 oxyethylene chains (Dutt 2004, Zhu et al., 1992; Zhu and Schelly 1992).

Figure 4 shows the emission spectra of 7H4MC in TX-100 reverse micelles at various $\mathrm{w}_{0}$ values. Quite surprisingly, in this reverse micelle at $\mathrm{w}_{0}=0$, in addition to the emission from the neutral form of the dye ( $370 \mathrm{~nm}$ ), another prominent band is observed around $450 \mathrm{~nm}$, which lies in the emission region of the anionic form. Interestingly, however, the intensity of this band significantly decreases on addition of water, instead of becoming more prominent. The absorption spectrum of 7H4MC in the "dry" TX-100 reverse micelles $\left(\mathrm{w}_{0}=0\right)$, also shows a shoulder band around $370 \mathrm{~nm}$ in addition to the main absorption band of the neutral form at $320 \mathrm{~nm}$ (Inset of Fig. 4), the former becoming quite weak on addition of water. The appearance of a band at longer wavelengths in the absorption and emission spectrum of 7H4MC in the "dry" TX-100 reverse micelles suggests that there is some specific interaction of the dye with the surfactant head groups, which is disrupted in the presence of water. As the TX-100 surfactant is reported to have a Lewis base character (Andrade and Costa, 2002), the longer wavelength absorption/emission bands are attributed to the formation of contact ion-pairs $\left(7 \mathrm{H} 4 \mathrm{MC}^{\delta-}: \mathrm{TX}-100^{\delta+}\right)$ at the interfacial region. With gradually increasing $\mathrm{w}_{0}$ value, the hydrophilic regions of TX-100 are hydrated and its Lewis basicity decreases. Consequently the formation of the contact ion-pairs of the dye with the oxyethylene chains $\left(7 \mathrm{H} 4 \mathrm{MC}^{\delta-}: \mathrm{TX}-100^{\delta+}\right)$, becomes disfavored.

As indicated from the emission spectra (cf. Fig. 4), with increasing $\mathrm{w}_{0}$ values in the TX-100 reverse micelles, the $450 \mathrm{~nm}$ band decreases in intensity and reaches a saturation, with the concomitant development of a new emission band around $490 \mathrm{~nm}$. The new emission band around $490 \mathrm{~nm}$, which appears above $\mathrm{w}_{0}=2$, is assigned to the tautomer form, $\mathrm{T}^{*}$, of the dye. The appearance of the $\mathrm{T}^{*}$ form beyond $\mathrm{w}_{0}=2$ is in accordance with previous propositions that in TX-100/benzene-hexane/water reverse micelles, water pool like structure starts forming around $\mathrm{w}_{0}=2$ 2.5 (Zhu and Schelly, 1992). Our results reveal that beyond $\mathrm{w}_{0}=2$, water molecules can reorient around 7H4MC to form a bridge between the $\mathrm{OH}$ and the $\mathrm{C}=\mathrm{O}$ groups, leading to the intramolecular proton transfer that yields the tautomeric species. The saturation in the emission intensity of the $450 \mathrm{~nm}$ band above $\mathrm{w}_{0} \sim 3$ indicates that the anionic form, $\mathrm{A}^{*}$, also partially contributes to this emission band at higher $\mathrm{w}_{0}$ values (in addition to the emission of the ion pair whose contribution is expected to decrease at higher $\mathrm{w}_{0}$ values). Nevertheless, it is quite clear that there is no appreciable formation of the anionic form of 


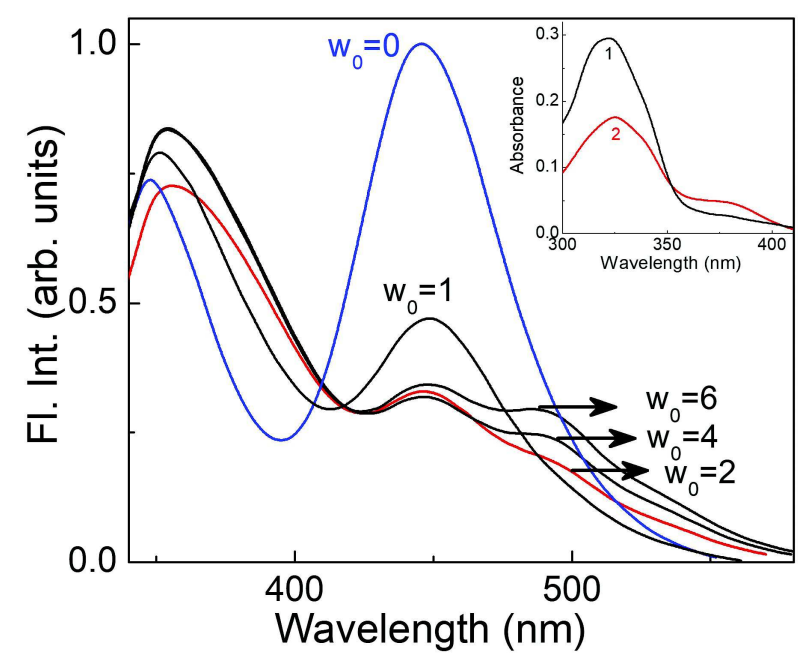

Fig. 4: Emission spectra of $7 \mathrm{H} 4 \mathrm{MC}$ in $\mathrm{TX}-100$ reverse micelles at different $w_{0}$ values. Excitation wavelength is $320 \mathrm{~nm}$. Inset shows the absorption spectra of $7 \mathrm{H} 4 \mathrm{MC}$ in $\mathrm{TX}-100$ reverse micelles at $\mathrm{w}_{0}=0$ (2) and $w_{0}=6$ (1). (Adapted with permission from Dutta Choudhury and Pal, 2009. Copyright 2009 American Chemical Society)

7H4MC in the nonionic TX-100 reverse micelles, unlike the ionic reverse micelles, BHDC and AOT. This difference cannot be merely attributed to the different charges of the surfactant head groups, since the anionic form of $7 \mathrm{H} 4 \mathrm{MC}$ is clearly observed in both anionic and cationic reverse micelles (cf. Fig. 2). The lower propensity for proton transfer in TX100 reverse micelles is rather considered to be due to its restricted aqueous microenvironment. As the water molecules are mainly dispersed near the hydrophilic oxyethylene chains of the TX-100 surfactants, and the water pool in the micellar core is not well formed, the ability of the confined water to accept a proton and solvate the dye anion at the interfacial region is considerably less. This makes the deprotonation of 7H4MC less favored in TX-100 than in AOT and BHDC reverse micelles (Dutta Choudhury and Pal, 2009).

\section{Excited State Prototropism of 7H4MC in IL- Water Mixtures}

Surface active ILs, especially those based on 1-alkyl3-methylimidazolium cations $\left(\mathrm{C}_{\mathrm{n}} \mathrm{mim}\right)$, are widely investigated due to the tunability of their amphiphilic character and aggregation behaviour by varying the alkyl chain length of the cation (Bowers et al., 2004;
Singh and Kumar 2007). Akin to conventional surfactants, the addition of surface active ILs to water, leads to the formation of micelle-like structures beyond a critical concentration of the IL (Bowers et al., 2004; Vanyúr et al., 2007; Wei et al., 2014). Furthermore, some recent studies have proposed that if water is added to the ILs, then initially reverse micelle-like structures are formed at lower water contents, with the creation of confined water pockets (Abe et al., 2014; Gao et al., 2016; Masaki et al., 2010; Saihara et al., 2015). Considering the high sensitivity of the probe dye, 7H4MC, to aqueous microenvironments, we have investigated the effect of small water additions on the excited state prototropism of the dye in IL-water mixtures of a series of 1-alkyl-3methylimidazolium cation $\left(\mathrm{C}_{\mathrm{n}} \mathrm{mim}\right)$ based ILs with varying alkyl chain lengths $(n=2,4,6,8$ and 10; $\mathrm{C}_{2} \mathrm{mim}, \mathrm{C}_{4} \mathrm{mim}, \mathrm{C}_{6} \mathrm{mim}, \mathrm{C}_{8} \mathrm{mim}$ and $\mathrm{C}_{10} \mathrm{mim}$, respectively), and having tetrafluoroborate, $\left[\mathrm{BF}_{4}\right]^{-}$as the common anion.

Our results revealed that in the pure ILs, $7 \mathrm{H} 4 \mathrm{MC}$ exists in the neutral form in the ground and excited states. However, on addition of water to the ILs, the excited neutral form of the dye is progressively converted to the anionic and the tautomeric species, leading to pronounced changes in the emission spectra. Representative emission spectra of 7H4MC in the $\left[\mathrm{C}_{10} \mathrm{mim}\right]\left[\mathrm{BF}_{4}\right] \mathrm{IL}$ at various $\mathrm{w}_{0}$ values are presented in Fig. 5A. It may be noted that the spectral features of $7 \mathrm{H} 4 \mathrm{MC}$ in the IL-water system are qualitatively similar to the cationic reverse micelle system, BHDC (cf. Fig. 2B) rather than the anionic reverse micelle AOT (cf. Fig. 2A) or organic solventwater mixtures (cf. spectra in dioxane-water mixture in Fig. 1). This indicates that the microenvironment around the dye in the IL-water mixtures are comparable to the microenvironment experienced by the dye in the BHDC reverse micelles. It was further observed that the relative contribution of the two prototropic species, $\mathrm{A}^{*}$ and $\mathrm{T}^{*}$, at a particular $\mathrm{w}_{0}$ value in the IL-water mixtures, gradually increases with the increasing alkyl chain length of the IL cation (Fig. $5 \mathrm{~B})$. Based on the observed results, it is confirmed that addition of water to the ILs leads to the organization of the surfactant-like IL cations in such a manner that the positively charged imidazolium head groups orient inwards to form a charged interface that surrounds and entraps the added water molecules, while the alkyl tails assemble and orient outward to 


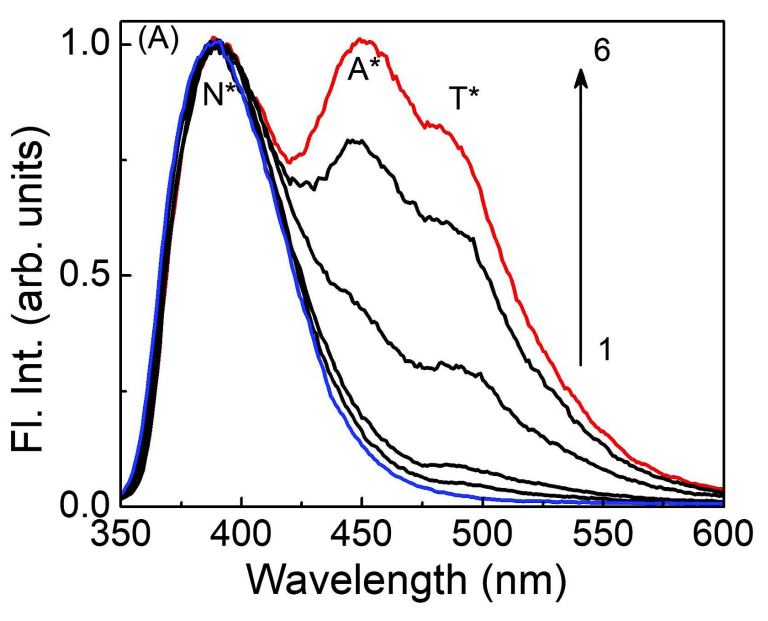

(B)
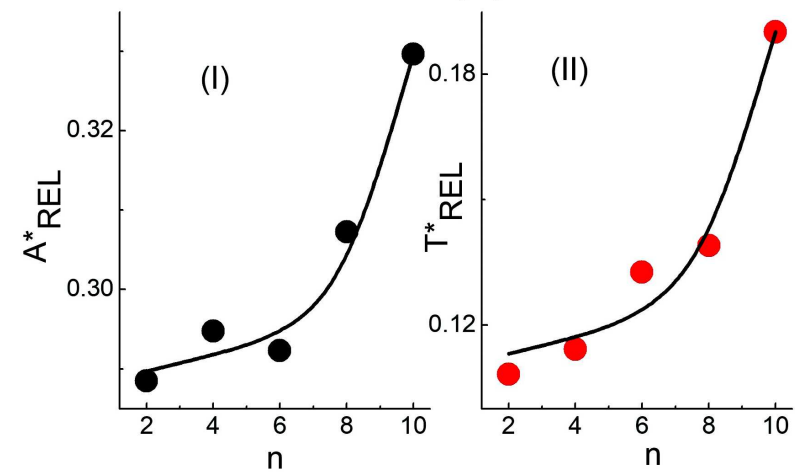

Fig. 5: (A) Emission spectra of $7 \mathrm{H} 4 \mathrm{MC}$ in $\left[\mathrm{C}_{10} \mathrm{mim}\right]\left[\mathrm{BF}_{4}\right]-$ water mixtures at $\mathrm{w}_{0}$ values of $0,0.6,1.3,1.8,2.5,2.8$ (1-6). Excitation wavelength is $320 \mathrm{~nm}$. (B) Relative emission contributions of the anionic $\left(A^{*}\right)$ and tautomeric $\left(T^{*}\right)$ forms of $7 \mathrm{H} 4 \mathrm{MC}$ in $\left[\mathrm{C}_{\mathrm{n}} \mathrm{mim}\right]\left[\mathrm{BF}_{4}\right]-$ water systems at $w_{0}=1.8$ with varying carbon atoms (n) in the alkyl chain of the IL cation. The lines are shown as a guide for the eyes

\section{References}

Abdel-Mottaleb M S A, El-Sayed B A, Abo-Aly M M and ElKady M Y (1989) Fluorescence Properties and Excited state Interactions of 7-hydroxy-4-methylcoumarin laser dye J Photochem Photobiol A 46 379-390

Abe H, Takekiyo T, Shigemi M, Yoshimura Y, Tsuge S, Hanasaki T, Ohishi K, Takata S and Suzuki J (2014) Direct Evidence of Confined Water in Room-Temperature Ionic Liquids by Complementary Use of Small-Angle X-ray and Neutron Scattering J Phys Chem Lett 5 1175-1180

Agazzi F M, Rodriguez J, Falcone R D, Silber J J and Correa N M (2013) PRODAN Dual Emission Feature To Monitor BHDC Interfacial Properties Changes with the External Organic Solvent Composition Langmuir 29 3556-3566

Amaro M, Brezovskyì J, Kovaìcovaì S, Syìkora J, Bednaìr D, form the nonpolar domain (cf. Chart 3). With increasing water content, the self-assembly of the IL medium progresses and the environment surrounding the $7 \mathrm{H} 4 \mathrm{MC}$ dye gradualy changes, which is reflected in the excited state prototropic equilibria and the emission spectra of the dye. The increase in the chain length of the ILs enhances their capability to form reverse-micelle like supramolecular assemblies with more well-defined water pools, and hence facilitates the prototropic transformation of 7H4MC.

In summary, this article provides a comprehensive overview of the excited state prototropic behavior of the probe dye, 7H4MC, in anionic, cationic and nonionic reverse micelles, and in the reverse micelle-like structures formed in IL-water mixtures at low water contents. The prototropic transformations of 7H4MC essentially reflect the different microenvironments experienced by the probe in these organized assemblies. These studies not only provide a fundamental understanding on proton transfer reactions but also help in gaining insights on the nature of the confined water in various biomimicking assemblies.

\section{Acknowledgment}

The authors gratefully acknowledge the generous support provided by the host institute, Bhabha Atomic Research Centre (BARC), Mumbai. We also thank all the co-authors of our published papers discussed in this article.

Nemec V, Liskovaì V, Kurumbang N P, Beerens K, Chaloupkovaì R, Paruch $\mathrm{K}$, Hof $\mathrm{M}$ and Damborskyì $\mathrm{J}$ (2015) Site-Specific Analysis of Protein Hydration Based on Unnatural Amino Acid Fluorescence J Am Chem Soc 137 4988-4992

Amdursky N, Simkovitch R and Huppert D (2014) ExcitedState Proton Transfer of Photoacids Adsorbed on Biomaterials J Phys Chem B 118 13859-13869

Andrade S M and Costa S M B (2002) The aqueous environment in AOT and Triton X-100 (w/o) microemulsions probed by fluorescence Photochem Photobiol Sci 1 500-506

Arnaut L G and Formosinho S J (1993) Excited-state proton transfer reactions I. Fundamentals and intermolecular reactions J Photochem Photobiol 75 1-20

Bardez E (1999) Excited-State Proton Transfer in Bifunctional 
Compounds Isr J Chem 39 319-332

Bardez E, Boutin P and Valeur B (1992) Photoinduced biprotonic transfer in 4-methylumbelliferone Chem Phys Lett 191 $142-148$

Blach D, Correa N M, Silber J J and Falcone R D (2011) Interfacial water with special electron donor properties: Effect of water-surfactant interaction in confined reversed micellar environments and its influence on the coordination chemistry of a copper complex J Colloid Int Sci 355 124130

Bowers J, Butts C P, Martin P J and Vergara-Gutierrez M C (2004) Aggregation Behavior of Aqueous Solutions of Ionic Liquids Langmuir 20 2191-2198

Chowdhury R, Saha A, Mandal A K, Jana B, Ghosh S and Bhattacharyya K (2015) Excited State Proton Transfer in the Lysosome of Live Lung Cells: Normal and Cancer Cells J Phys Chem B 119 2149-2156

Cohen B, Álvarez C M, Carmona N A, Organero J A and Douhal A (2011) Proton-Transfer Reaction Dynamics within the Human Serum Albumin Protein J Phys Chem B 1157637 7647

de Melo J S and Maçanita A L (1993) Three Interconverting excited species: experimental study and solution of the general photokinetic triangle by time-resolved studies Chem Phys Lett 204 556-562

DeSilva N, Minezawa N and Gordon M S (2013) Excited-State Hydrogen Atom Transfer Reaction in Solvated 7-Hydroxy4-methylcoumarin J Phys Chem B 117 15386-15394

Douhal A, Angulo G, Gil M, Organero J Á, Sanz M and Tormo L (2007) Observation of Three Behaviors in Confined Liquid Water within a Nanopool Hosting Proton-Transfer Reactions J Phys Chem B 111 5487-5493

Dutt G B (2004) Does the Onset of Water Droplet Formation Alter the Microenvironment of the Hydrophobic Probes Solubilized in Nonionic Reverse Micelles? J Phys Chem B 108 7944-7949

Dutta Choudhury S and Pal H (2009) Modulation of ExcitedState Proton-Transfer Reactions of 7-Hydroxy-4methylcoumarin in Ionic and Nonionic Reverse Micelles $J$ Phys Chem B 113 6736-6744

Dutta Choudhury S, Nath S and Pal H (2008) Excited-State Proton Transfer Behavior of 7-Hydroxy-4-methylcoumarin in AOT Reverse Micelles J Phys Chem B 112 7748-7753

Formosinho S J and Arnaut L G (1993) Excited-state proton transfer reactions II. Intramolecular reactions $J$ Photochem Photobiol A 75 21-48

Gao J and Wagner N J (2016) Water Nanocluster Formation in the Ionic Liquid 1-Butyl-3-methylimidazolium Tetrafluoroborate $\left(\left[\mathrm{C}_{4} \mathrm{mim}\right]\left[\mathrm{BF}_{4}\right]\right)-\mathrm{D}_{2} \mathrm{O}$ Mixtures Langmuir 32 5078-5084

Georgieva I, Trendafilova N, Aquino A J A and Lischka H (2007) Excited-State Proton Transfer in 7-Hydroxy-4methylcoumarin along a Hydrogen-Bonded Water Wire $J$ Phys Chem A 111 127-135

Gepshtein R, Leiderman P, Huppert D, Project E, Nachliel E and Gutman M (2006) Proton Antenna Effect of the $\gamma$ Cyclodextrin Outer Surface, Measured by Excited State Proton Transfer J Phys Chem B 110 26354-26364

Jain T K, Varshney M and Maitra A (1989) Structural studies of Aerosol OT reverse micellar aggregates by FT-IR spectroscopy $J$ Phys Chem 93 7409-7416

Kalyanasundaram, K (1987) Photochemistry in Microheterogeneous Systems, Academic Press

Kwon J E and Park S Y (2011) Advanced Organic Optoelectronic Materials: Harnessing Excited-State Intramolecular Proton Transfer (ESIPT) Process Adv Mater 23 3615-3642

Luisi P L, Giomini M, Pileni M P and Robinson B H (1988) Reverse micelles as hosts for proteins and small molecules Biochim Biophys Acta 947 209-246

Masaki T, Nishikawa K and Shirota H (2010) Microscopic Study of Ionic Liquid- $\mathrm{H}_{2} \mathrm{O}$ Systems: Alkyl-Group Dependence of 1-Alkyl-3-Methylimidazolium Cation J Phys Chem B $1146323-6331$

McNeil R and Thomas J K (1981) Benzylhexadecyldimethylammonium Chloride in Microemulsions and Micelles J Colloid Int Sci 83 57-65

Moilanen D E, Levinger N E, Spry D B, Fayer M D (2007) Confinement or the Nature of the Interface? Dynamics of Nanoscopic Water J Am Chem Soc 129 14311-14318

Moriya T (1983) Excited-state Reactions of Coumarins in Aqueous Solutions. I. The Phototautomerization of 7Hydroxycoumarin and Its Derivative Bull Chem Soc Jpn 56 6-14

Piletic I R, Moilanen D E, Spry D B, Levinger N E and Fayer M D (2006) Testing the Core/Shell Model of Nanoconfined Water in Reverse Micelles Using Linear and Nonlinear IR Spectroscopy J Phys Chem A 110 4985-4999

Saihara K, Yoshimura Y, Ohta S and Shimuzu A (2015) Properties of water confined in ionic liquids Sci Rep 510619

Simkovitch R, da Silva L P, da Silva J C G E and Huppert D (2016) Comparison of the Photoprotolytic Processes of Three 7-Hydroxycoumarins J Phys Chem B $12010297-$ 10310

Singh T and Kumar A(2007) Aggregation Behavior of Ionic Liquids 
in Aqueous Solutions: Effect of Alkyl Chain Length, Cations, and Anions J Phys Chem B 111 7843-7851

Singhal N, Mishra A and Datta A (2016) Excited-State Proton Transfer and Conformational Relaxation of 2-(42 Pyridyl)benzimidazole in Nafion Films Chem Phys Chem 17 3004-3009

Siwick B J and Bakker H (2007) On the Role of Water in Intermolecular Proton-Transfer Reactions $\mathrm{J} \mathrm{Am} \mathrm{Chem} \mathrm{Soc}$ 129 13412-13420

Takeuchi S and Tahara T (2007) The answer to concerted versus step-wise controversy for the double proton transfer mechanism of 7-azaindole dimer in solution Proc Natl Acad Sci 104 5285-5290

Vanyúr R, Biczók L and Miskolczy Z (2007) Micelle formation of 1-alkyl-3-methylimidazolium bromide ionic liquids in aqueous solution Colloids Surf A 299 256-261

Waluk J (2003) Hydrogen-Bonding-Induced Phenomena in Bifunctional Heteroazaaromatics Acc Chem Res 36 832838
Wei Y, Wang F, Zhang Z, Ren C and Lin Y (2014) Micellization and Thermodynamic Study of 1-Alkyl-3methylimidazolium Tetrafluoroborate Ionic Liquids in Aqueous Solution J Chem Eng Data 59 1120-1129

Zhao J, Ji S, Chen Y, Guo H and Yang P (2012) Excited state intramolecular proton transfer (ESIPT): from principal photophysics to the development of new chromophores and applications in fluorescent molecular probes and luminescent materials Phys Chem Chem Phys 14 88038817

Zhu D-M and Schelly Z A (1992) Investigation of the micropolarities in reverse micelles of Triton X-100 in mixed solvents of benzene and n-hexane J Phys Chem 96 71217126

Zhu D-M, Wu X and Schelly Z A (1992) Reverse micelles and water in oil microemulsions of Triton X 100 in mixed solvents of benzene and n-hexane. Dynamic light scattering and turbidity studies Langmuir 8 1538-1540. 